\title{
Synchrotron Jet Model for the 1989-1996 Cycle of Activity of NGC 4151
}

\author{
I.I. Pronik \\ Crimean Astrophysical Observatory, p. Nauchny, 98409 Crimea, \\ Ukraine
}

\begin{abstract}
Observational data from the X-ray through the $U V$ and optical of variations of the NGC 4151 nucleus were compiled. The characteristics of the variability related to the 1989-1996 cycle of activity show remarkable similarity to those of blazars and do not contradict the supposition that this cycle of activity was caused by a synchrotron jet (SJ). Some of the activity of previous cycles were interpreted in the framework of a SJ model and some of that of a disc instability model (DI). We ask the question if different cycles of activity can be of a different nature caused by a different accretion rate.
\end{abstract}

Several cycles of activity in optical of the NGC 4151 nucleus were observed over 1906-1997. As SJ so DI models were proposed for their explaining. 19891997 cycle activity was observed the most tightly by many authors. They supposed that this cycle was connected with a new variable source of high ultraviolet emission and very high degree variations: the $U V$ flux $(1440 \AA)$ of new variable source in 1993 was by a factor 5 more than in 1984-1985.

Compiled from literature data of continuum flux variability in the X-ray, UV bands and optical showed that they exhibit characteristics of blazar variability:

1. Variability is present on time scales from minutes up to 7 years. There is a strong consensus that microvariability on time-scales of a few hours is important sign of blazers.

2. Amplitude of the continuum variations decreases with wavelength increasing. During 7.3 years in 1989-1997 amplitudes of variability in diaphragm $20^{\prime \prime}$ are equal to $2 .^{m} 0,1 .^{m} 5,0 .^{m} 9,0 .^{m} 8$ and $0 .^{m} 7$ in the $U B V R I$ bands, respectively.

3. Flares play an important role in general brightening of the NGC 4151 nucleus. The rate of the energy output in the $U$ band during the flares on timescale 10-20 days was more than a magnitude higher than those on timescale years.

4. Continuum varies in phase within 1 day at all wavelengths from $\mathrm{X}$-ray to optical. Observations during 10 days in December 1993 showed that upper limit to the lags are 0.15 day between $1275 \AA$ and the other ultraviolet bands, less than 0.3 day between $1275 \AA$ and $1.5 \mathrm{keV}$, and less than 1 day between $1275 \AA$ and $5125 \AA$. According to observations in 1989-1996 time delay between $3600 \AA$ and $5300 \AA$ at a significance level of $99 \%$ is less than 0.8 day.

5. Energy distribution at flux excesses during the 1.5-2.0 years variations and during the 10-150 days flares in the $U V$ and optical regions showed a power 
law form: $\mathrm{F}_{\nu} \sim \nu^{\alpha}$. For $1250-3200 \AA \alpha=-1.2$; in the range $3600-8300 \AA$ for flares $+0.18 \geq \alpha \geq-1.83$, for smooth light curve: $-0.33 \geq \alpha \geq-1.30$.

6. Strong variability of total flux and of spectral index $\alpha$ in the $U V$ and optical: for $1250-3200 \AA \Delta \alpha=1.4$; in the range $3600-8300 \AA$ for flares $\Delta \alpha=2.01$, for the smooth light curve: $\Delta \alpha=0.97$. Reduced to aperture $\mathrm{D}=5^{\prime \prime}$, amplitude of the $U$ flux variation was a factor more than 50 and of the $V$ flux variations was a factor more than 9 .

7. There was a flattening of the spectrum of variable flux in the range $1250-8300 \AA$ with the brightening as for flares so for smooth light curves.

8. The Structure Function $(\mathrm{SF})$ parameter $\mathrm{b}=\mathrm{d} \log (\mathrm{SF}) / \mathrm{d} \log (\mathrm{dt})$ was obtained for the $U B V R I$ flux variations. It was shown that the highest values of SF slopes "b" during the beginning of active period in 1989-1990 were in the range $0.8 \leq \mathrm{b} \leq 1.1$. For $10-150$ day flares slope " $\mathrm{b}$ " was equal up to 2.2 exhibiting the extreme shot-noise process characteristic for radio variable flux of blazers.

Obtained characteristics of variable source acted in the NGC 4151 nucleus in 1989-1997 do not contradict of the SJ model when increasing of the nucleus brightening is caused by the clouds of synchrotron radiation ejected from the nucleus during its active period. The general brightening of the nucleus can be caused by accumulation of such clouds. The simplest model for the variability is an adiabatically expanding sphere of magnetized relativistic electrons as it was considered by Shklovsky (1960), van der Laan (1966), Begelman et al. (1984), and others. Flux density $\mathrm{S}_{\nu}$ of optically thick cloud with the conserved magnetic field $\mathrm{B}$ at a frequency $\nu$ will be $\mathrm{S}_{\nu} \sim \nu^{5 / 2} \mathrm{~B}^{-1 / 2}$ and of optically thin cloud will be $\mathrm{S}_{\nu} \sim \nu^{-\alpha} \mathrm{B}^{\alpha+1}$.

Spectral index $\alpha$ of flare emission increased from the beginning $(\alpha=-1.8)$ to the end of observational period (through $\alpha=-0.5$ to $\alpha=+0.18$ ). It evidenced that optical thickness of synchrotron clouds increased. At the beginning of the nucleus brightening the clouds were optically thin and at the end of the nucleus brightening their emission became optically thick. Low envelope of the $U B V R I$ light curves represents the resulting emission of accumulated clouds of different optical thickness. Increasing of spectral index with time from -1.30 up to -0.33 can be connected with increasing of percentage of optically thick clouds inside the accumulated ensemble of clouds.

Nonthermal anisotropic radio emission of the NGC 4151 nucleus at $\lambda 6$ $\mathrm{cm}$ and $20 \mathrm{~cm}$ was discovered by Jonston et al. (1982). Variable source of synchrotron optical emission in the nucleus was suspected by Thompson, Landstreet et al., (1979) and Schmidt, Miller (1980). They have obtained that the continuum polarization of variable source of NGC 4151 increased smoothly from a value of $1.2 \%$ in the red to $\sim 2.5 \%$ at $\lambda 4000 \AA$. Qualitatively the same wavelength dependence observed in other polarized Seyferts. There was observed significant variations in the degree of polarization between 1975 April and 1977 May.

Lyuty and Doroshenko (1999) discussed (1968-1983) and (1990-1998) cycles of the NGC 4151 nucleus activity in frame of DI model. We put a question can different cycles of activity have different nature caused by different accretion rate. 